OPEN ACCESS

Edited by:

Barbara Ambrose,

New York Botanical Garden,

United States

Reviewed by:

Petr Smýkal,

Palacký University, Olomouc, Czechia

Raquel Iglesias Fernández,

Polytechnic University of

Madrid, Spain

Zhongsong Liu,

Hunan Agricultural University, China Martin Sagasser,

University of Bielefeld, Germany

${ }^{*}$ Correspondence:

Enrico Magnani

enrico.magnani@inra.fr

Specialty section:

This article was submitted to

Plant Development and EvoDevo,

a section of the journal

Frontiers in Plant Science

Received: 08 October 2019 Accepted: 23 December 2019

Published: 06 February 2020

Citation:

Coen O, Lu J, Xu W, Pateyron S, Grain $D$, Péchoux $C$, Lepiniec $L$ and Magnani E (2020) A TRANSPARENT

TESTA Transcriptional Module Regulates Endothelium Polarity.

Front. Plant Sci. 10:1801.

doi: $10.3389 /$ fpls.2019.01801

\section{A TRANSPARENT TESTA Transcriptional Module Regulates Endothelium Polarity}

\author{
Olivier Coen ${ }^{1,2}$, Jing Lu ${ }^{1,2}$, Wenjia Xu ${ }^{1}$, Stéphanie Pateyron ${ }^{3}$, Damaris Grain ${ }^{1}$, \\ Christine Péchoux ${ }^{4}$, Loïc Lepiniec ${ }^{1}$ and Enrico Magnani ${ }^{1 \text { * }}$
}

1 Institut Jean-Pierre Bourgin, INRA, AgroParisTech, CNRS, University of Paris-Saclay, Versailles, France, ${ }^{2}$ École Doctorale 567 Sciences du Végétal, University Paris-Sud, University of Paris-Saclay, Orsay, France, ${ }^{3}$ TranscriptOmic Platform of IPS2, Institute of Plant Sciences Paris Saclay IPS2, CNRS, INRA, Université Paris-Sud, Université Evry, Université Paris-Saclay, Orsay, France, ${ }^{4}$ INRA, Génétique Animale et Biologie Intégrative, Jouy-en-Josas, France

Seeds have greatly contributed to the successful colonization of land by plants. Compared to spores, seeds carry nutrients, rely less on water for germination, provide a higher degree of protection against biotic and abiotic stresses, and can disperse in different ways. Such advantages are, to a great extent, provided by the seed coat. The evolution of a multi-function seed-coat is inheritably linked to the evolution of tissue polarity, which allows the development of morphologically and functionally distinct domains. Here, we show that the endothelium, the innermost cell layer of the seed coat, displays distinct morphological features along the proximal-distal axis. Furthermore, we identified a TRANSPARENT TESTA transcriptional module that contributes to establishing endothelium polarity and responsiveness to fertilization. Finally, we characterized its downstream gene pathway by whole-genome transcriptional analyses. We speculate that such a regulatory module might have been responsible for the evolution of morphological diversity in seed shape, micropylar pore formation, and cuticle deposition.

Keywords: seed coat, endothelium, polarity, TRANSPARENT TESTA, fertilization

\section{INTRODUCTION}

The seed coat consists of cell layers that surround, nourish, protect, and facilitate the dispersal of the fertilization product(s) (Coen and Magnani, 2018). As a whole, the seed coat is a highly polar structure displaying morphologically and functionally distinct domains responsible for different seed functions (Haughn and Chaudhury, 2005; Coen and Magnani, 2018). Along the seed mediallateral polarity axis, different cell layers follow unique differentiation pathways. Furthermore, cellpatterning along the proximal-distal axis defines the chalazal and micropylar regions (Figure 1A). Finally, integument number and cell thickness deeply affect seed curvature along the adaxialabaxial axis.

In Arabidopsis, the seed coat comprises an outer (oi) and an inner (ii) integument (Schneitz et al., 1995). Both ii and oi initiate in the ovule as two-cell layered primordia (iil or endothelium, ii2, oi1, and oi2) that grow by anticlinal cell divisions to surround the female gametophyte. At the end of 

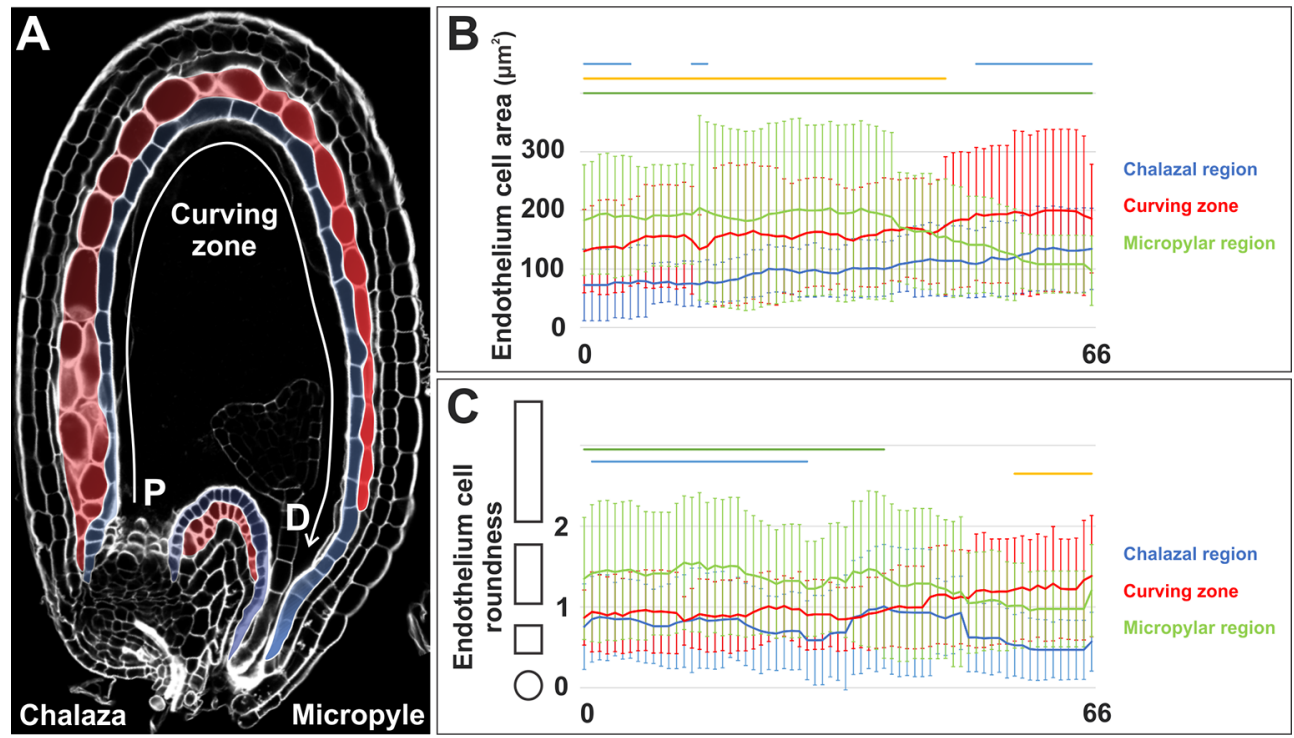

FIGURE 1 | The endothelium proximal-distal polarity axis. (A) Longitudinal mid-plane of a wild type seed at 6 DAF. The endothelium is highlighted in blue whereas ii1' and ii1" cells are highlighted in red. P, proximal; D, distal. (B, C) Average endothelium cell area in $\mu \mathrm{m}^{2} \mathbf{( A )}$ and cell roundness (B) (see Methods) along the proximal-distal axis of the chalazal, curving zone, and micropylar regions (arbitrarily divided in 67 points) as observed in longitudinal mid-planes of wild type seeds at 4 DAF. The shapes on the left of the graph in panel B exemplify how cell shape changes along the $y$-axis. Lines at the top of the graph indicate regions of statistically significant difference between chalazal region and curving zone (green), chalazal and micropylar regions (yellow), and curving zone and micropylar region (red) (twotailed Student's t-test; $P<0.05)$. Error bars indicate standard deviation $(n=33)$. Ecotype Ws.

ovule development, periclinal cell divisions of the endothelium, the innermost integument cell layer, give rise to one or two subepidermal cell layers (ii1' and ii1") (Figure 1A) (Schneitz et al., 1995; Debeaujon et al., 2003; Coen et al., 2017). Furthermore, a fraction of ovules displays additional sub-epidermal cell strings (oi1'), of chalazal origin, in between oil and oi2 (Fiume et al., 2017a). Double fertilization of the female gametophyte gives rise to embryo and endosperm and marks the transition from ovule to seed (Schneitz et al., 1995). The seed coat does not actively participate in the fertilization process but undergoes cell expansion and differentiation in coordination with the fertilization products (Ingram, 2010).

The endothelium lies at the interface between maternal tissues and fertilization products and displays unique biological properties. On the one hand, it shows remarkable developmental plasticity in interplaying with the endosperm to orchestrate seed growth (Ingram, 2010). On the other hand, it becomes a highly differentiated tissue. A cutin-based apoplastic barrier is deposited on the adaxial side of the endothelium, toward the endosperm, during ovule development, and in response to fertilization (De Giorgi et al., 2015; Loubery et al., 2018; Coen et al., 2019). Furthermore, fertilization triggers the accumulation in the endothelium of proanthocyanidins (PAs), flavonoid compounds that give the characteristic brown color to Arabidopsis seeds (Lepiniec et al., 2006). Genetic screens have identified several mutants defective in PA accumulation, collectively named transparent testa $(t t)$. TT loci are involved in different aspects of tannin deposition: biosynthesis, transport, and regulation
(Koornneef, 1990; Lepiniec et al., 2006). A handful of genes have been also found to regulate ii development. The MADS box transcription factor TT16 not only regulates PAs accumulation and cutin deposition but also endothelium cell expansion and orientation (Nesi et al., 2002; Ehlers et al., 2016; Coen et al., 2017; Coen et al., 2019). Furthermore, TT16 works redundantly with another MADS box transcription factor, SEEDSTICK (STK), to initiate endothelium periclinal cell divisions (Mizzotti et al., 2012; Coen et al., 2017) and oppositely to SHATTERPROOF 1 and 2 MADS box transcription factors to establish iil' proximal-distal polarity (Ehlers et al., 2016). The WRKY transcription factor TRANSPARENT TESTA GLABRA 2 (TTG2) coordinates integuments and endosperm growth (Garcia et al., 2005). ttg2 mutant seeds show premature arrest of endosperm development and reduced seed size. Finally, the TT1 C2H2 zinc-finger transcription factor plays a role in PAs deposition and cuticle biosynthesis but there have been conflicting reports concerning its role in regulating endothelium cell shape (Sagasser et al., 2002; Debeaujon et al., 2003; Appelhagen et al., 2011; Coen et al., 2019).

Here we show that endothelium development is modulated along the proximal-distal axis. Our expression and genetic analyses indicate a role for TT1 in endothelium and ii1' polar cell patterning. Furthermore, we tested TT1 genetic interaction with all known regulators of endothelium development and placed TT1 downstream of TT16 in the development of the proximal region of the inner integument. Finally, transcriptomic analyses of an inducible form of TT1 revealed its downstream target genes. 


\section{METHODS}

\section{Plant Material}

Arabidopsis thaliana plants of ecotype Columbia (Col-0) or Wassilewskija (Ws-2) were used as wild type controls as appropriate. The tt16-1 mutant was isolated in the Ws- 2 accession and then backcrossed to the Col-0 accession more than three times (Nesi et al., 2002; Xu et al., 2016). tt1-3, stk-2, shp1-1;shp2-1, and map18/pcap2 mutants are in the Col-0 accession (Liljegren et al., 2000; Pinyopich et al., 2003; Appelhagen et al., 2011; Kato et al., 2019). tt1-4 mutant is in the Ws-2 accession (Brunaud et al., 2002). ProML1:gML1$m$ Citrine; $m l 1-3$ line is in the Col-0 accession (Meyer et al., 2017).

Days after flowering were counted starting from the emergence of the pistil from closed flowers; 0 DAF equals stage 3-V of ovule development (Schneitz et al., 1995).

\section{Transgenic Plants}

The Agrobacterium tumefaciens strain C58C1 was used to stably transform Arabidopsis plants using the floral dip method (Clough and Bent, 1998). Transformants were selected on MS medium containing hygromycin $\left(50 \mathrm{mg} \mathrm{L}^{-1}\right)$ and subsequently transferred to soil for further characterization.

\section{Expression Analysis by Quantitative PCR}

Ovules and seeds used for total RNA extraction were frozen in liquid nitrogen immediately after harvest and stored at $-80^{\circ} \mathrm{C}$ prior to extraction. Four independent biological samples were used for each analysis. Each replicate comprised the content in ovules/seeds of 10 to 15 pistil/siliques. Total RNA was extracted using the RNeasy Mini kit (Qiagen), including RNase-Free DNase Set (Qiagen) treatment during washing, according to the manufacturer's instructions, and subsequently stored at $-80^{\circ}$ C. The Superscript Reverse Transcriptase II kit (Invitrogen) was used to generate cDNA from $1 \mu \mathrm{g}$ of total RNA. Each cDNA sample was diluted 1:125 in water. Quantitative PCRs were performed with the SYBR Green kit (Bio-Rad) on a Bio-Rad CFX real-time PCR machine. For each reaction, $4.4 \mu \mathrm{L}$ of diluted cDNA were added to $5 \mu \mathrm{l}$ of SYBR Green and to $0.3 \mu \mathrm{l}$ of each primer $(10 \mu \mathrm{M})$ (Supplemental Table 2). Melt curves have been performed and primer efficiency has been tested (primers with efficiency between $85 \%$ and $100 \%$ have been used in this study). Expression levels were first normalized by the geometrical mean of the expression levels of four reference genes (GAPDH, AT4G12590, AT4G02080, and AT3G25800) (Dekkers et al., 2012), and subsequently normalized by the expression level of the adequate control. Means and standard deviations were calculated from four independent biological samples.

\section{Cloning and Construction}

PCR amplification of 3kbProTT1:gTT1 was performed from Arabidopsis (Col-0) genomic DNA (https://www.ncbi.nlm.nih. gov/gene/840386) using the gene-specific primers (5' GGGGACAAGTTTGTACAAAAAAGCAGGCTCTAACCA TTTGCTTGTGTCAACA -3') and (5'- GGGGACCACTTTGT ACAAGAAAGCTGGGTCAAAAAACAAAGTCTCGGAGAC AG $-3^{\prime}$ ) carrying the attB1 and attB2 Gateway recombination sites. 3kbProTT1:gTT1 was recombined into the pDONR207 vector (Gateway recombination). Compared to what annotated on the TAIR website (www.arabidopsis.org), the 3kbProTT1:gTT1 DNA fragment misses an adenine in position -1250 of the promoter and the CATATATATATATATATATATATATATA TATATA sequence in position $605-638$ of the intron. PCR amplification of $g T T 1$ was performed using the gene-specific primers (5' - GGGGACAAGTTTGTACAAAAAAGCAGGC TCAATGGAGTCACCACCACTATACGAGA -3') and (5'GGGGACCACTTTGTACAAGAAAGCTGGGTCAAA AAACAAAGTCTCGGAGACAGAT -3') carrying the attB1 and attB2 Gateway recombination sites. For GR-inducible analyses, the gTT1 sequence was recombined (Gateway recombination) into the $p R 1 R 2 \Delta G R$ binary vector (Baudry et al., 2004).

\section{Microscopy}

Prior to microscopy analyses, siliques were dissected and septums (containing seeds) were harvested.

Calcofluor M2R white (fluorescent brightener 28; Sigma Aldrich) analyses were conducted as previously described (Coen et al., 2019). GFP and citrine expressing lines were analyzed 1 hour after mounting in a Propidium iodide $(100 \mu \mathrm{g}$ $\left.\mathrm{mL}^{-1}\right)$, sucrose $(7 \%)$ solution, as previously described (Figueiredo et al., 2016). mPS-PI samples were prepared as previously described (Xu et al., 2016). Transmission electron microscopy analyses were conducted as previously described (Coen et al., 2019).

Modified pseudo-Schiff propidium iodide (mPS-PI) or calcofluor stained and GFP or citrine fluorescent samples were analyzed with a Leica TCS-SP5 or Leica TCS-SP8 spectral confocal laser scanning microscope (Leica Microsystems). For TEM, samples were examined with Hitachi HT7700 electron microscope operated at $80 \mathrm{kV}$ (Elexience-France), and images were acquired with a charge-coupled device camera (AMT). For confocal microscopy, pictures showing mid-plane longitudinal sections of seeds were captured when possible. Otherwise, three dimensional z-stacks were acquired, and the mid-plane longitudinal sections were obtained with the Volume Viewer plugin of the Image J software (Schneider et al., 2012).

\section{Quantitative Morphological Analyses}

Quantitative analyses of cell area and roundness were conducted as previously described (Coen et al., 2017). Ratio between endothelium anticlinal and periclinal cell length was obtained by assimilating endothelium cells to rectangles (in first approximation), and calculating their growth polarity (GP) as $\mathrm{GP}=\frac{1}{2}\left(\frac{P}{L}-2\right)$ where $P$ and $L$ represent perimeter and periclinal length of the cell, respectively. In the case of a perfect rectangle, GP represents the ratio between anticlinal and periclinal lengths.

\section{Transcriptome Studies}

Six Col-0 and Pro35S:gTT1-GR main inflorescences at stage 6.50 (Boyes et al., 2001) were harvested and immersed in a Silwet $0.005 \%$ and $50 \mu \mathrm{M}$ cycloheximide (CHX) solution, vacuum treated for 30 minutes, and incubated for 1 hour at room temperature. A dexamethasone (DEX) solution in ethanol was added to three Col-0 and Pro35S:gTT1-GR CHX-treated 
inflorescences to a final concentration of $50 \mu \mathrm{M}$. The same amount of ethanol without DEX was added to the remaining three Col-0 and Pro35S:gTT1-GR CHX-treated inflorescences. Finally, the inflorescences were vacuum treated for 30 minutes, incubated for 1 hour at room temperature, and frozen individually in liquid nitrogen. Total RNA was extracted using Ambion Mirvana miRNA kit, followed by the Ambion TURBO DNAse kit, according to the supplier's instructions. The RNA integrity number was higher than eight. Microarray analysis was carried out using the CATMAv7 array (Lurin et al., 2004) based on AGILENT technology. Three independent biological replicates were produced. For each comparison, one technical replicate with fluorochrome reversal was performed for each biological replicate (i.e. four hybridizations per comparison). The labeling of cRNAs with Cy3-dUTP or Cy5-dUTP was performed as described in Two-Color Microarray-Based Gene Expression Analysis Low Input Quick Amp Labeling manual (Agilent Technologies, Inc.). The hybridization and washing were performed according to Agilent Microarray Hybridization Chamber User Guide instructions (Agilent Technologies, Inc.). Two micron scanning was performed with InnoScan900 scanner (Innopsys, Carbonne, France) and raw data were extracted using Mapix software (Innopsys, Carbonne, France).

\section{Statistical Analysis of Microarray Data}

For each array, the raw data comprised the logarithm of median feature pixel intensity at wavelengths $635 \mathrm{~nm}$ (red) and $532 \mathrm{~nm}$ (green). For each array, a global intensity-dependent normalization using the loess procedure (Yang et al., 2002) was performed to correct the dye bias. The differential analysis is based on the log-ratios averaging over the duplicate probes and over the technical replicates. Hence the numbers of available data for each gene equals the number of biological replicates and are used to calculate the moderated t-test (Smyth, 2004). Under, the null hypothesis, no evidence that the specific variances vary between probes is highlighted by Limma and consequently the moderated t-statistic is assumed to follow a standard normal distribution. To control the false discovery rate, adjusted pvalues found using the optimized FDR approach of (Storey and Tibshirani, 2003) were calculated. We considered as being differentially expressed the probes with an adjusted p-value $\leq$ 0.05. The analysis was done with the R software (http://www.Rproject.org). The function SqueezeVar of the Limma library was used to smooth the specific variances by computing empirical Bayes posterior means. The library kerfdr was used to calculate the adjusted $\mathrm{p}$-values.

\section{Gene Ontology Analyses}

Gene ontology annotation analyses were conducted on the TAIR website (www.arabidopsis.org). GO enrichment analyses were conducted using the gene ontology enrichment analysis and visualization tool (Eden et al., 2009).

\section{Data Deposition}

Microarray data from this article were deposited in the international repository GEO, Gene Expression Omnibus (Edgar et al., 2002), accession number: GSE 134014. All steps of the experiment, from growth conditions to bioinformatic and statistical analyses, were detailed in CATdb (Gagnot et al., 2008) (Project: RA15-05_TT1) according to the "Minimum Information About a Microarray Experiment” standards.

\section{Accession Numbers}

Sequence data from this article can be found in the GenBank/ EMBL data libraries under the following accession numbers: TT16 (AT5G23260), TT1 (AT1G34790), SHP1 (AT3G58780), SHP2 (AT2G42830), STK (AT4G09960), ML1 (AT4G21750), PDF2 (AT4G04890), CR4 (AT3G59420), TT2 (AT5G35550), TT8 (AT4G09820), TTG1 (AT5G24520), TTG2 (AT2G37260), FIE (AT3G20740), and MSI1 (AT5G58230). Supplemental Table 1 lists the accessions numbers for the genes detected by microarray analyses.

\section{RESULTS}

\section{The Endothelium Displays a Proximal- Distal Polarity Axis}

To determine if the endothelium displays morphological differences along the proximal distal axis (Figure 1A), we analyzed seeds using the modified pseudo-Schiff propidium iodide (mPS-PI) and quantified cellular parameters in the chalazal, curving zone and micropylar regions (Figure 1A, see Methods). The chalazal region of the endothelium was characterized by smaller and rounder cells, when compared to the other regions (Figures 1B, C). Micropylar endothelium cells changed from relatively large and elongated to relatively small and round along the proximal-distal axis (Figures 1B, C). Finally, the endothelium curving zone showed cellular parameters intermediate between the chalazal and micropylar regions (Figures $\mathbf{1 B}, \mathbf{C}$ ). This analysis indicate that the endothelium displays a proximal distal polarity axis.

\section{TRANSPARENT TESTA 1 Regulates the Polarity of Endothelium Cell Expansion}

TT16 and TT1 are the only two genes that have been found to regulate endothelium cell patterning (Nesi et al., 2002; Sagasser et al., 2002; Ehlers et al., 2016; Coen et al., 2017). Nevertheless, the role of TT1 in endothelium development is still controversial. Qualitative analyses of endothelium cell morphology in $t$ t 1 mutant seeds by differential interference contrast microscopy gave conflicting results. $t t 1$ endothelium cells have been indeed reported as irregular in shape (Sagasser et al., 2002) or wild type looking (Appelhagen et al., 2011). To test if TT1 plays a role in endothelium cell patterning, we analyzed $t t 1$ mutant ovules and seeds by mPS-PI or calcofluor staining imaging techniques as well as by transmission electron microscopy. Whereas $t t 1$ and wild type ovules appeared indistinguishable, longitudinal midplanes of $t t 1$ seeds at $4 \mathrm{DAF}$ exhibited anticlinally over-elongated endothelium cells at the chalazal region and periclinally overexpanded endothelium cells on their adaxial side, leaning on the embryo, at the micropylar region, when compared to the wild type (Figures 2A, C, G, H, and Supplemental Figure 1). At 6 

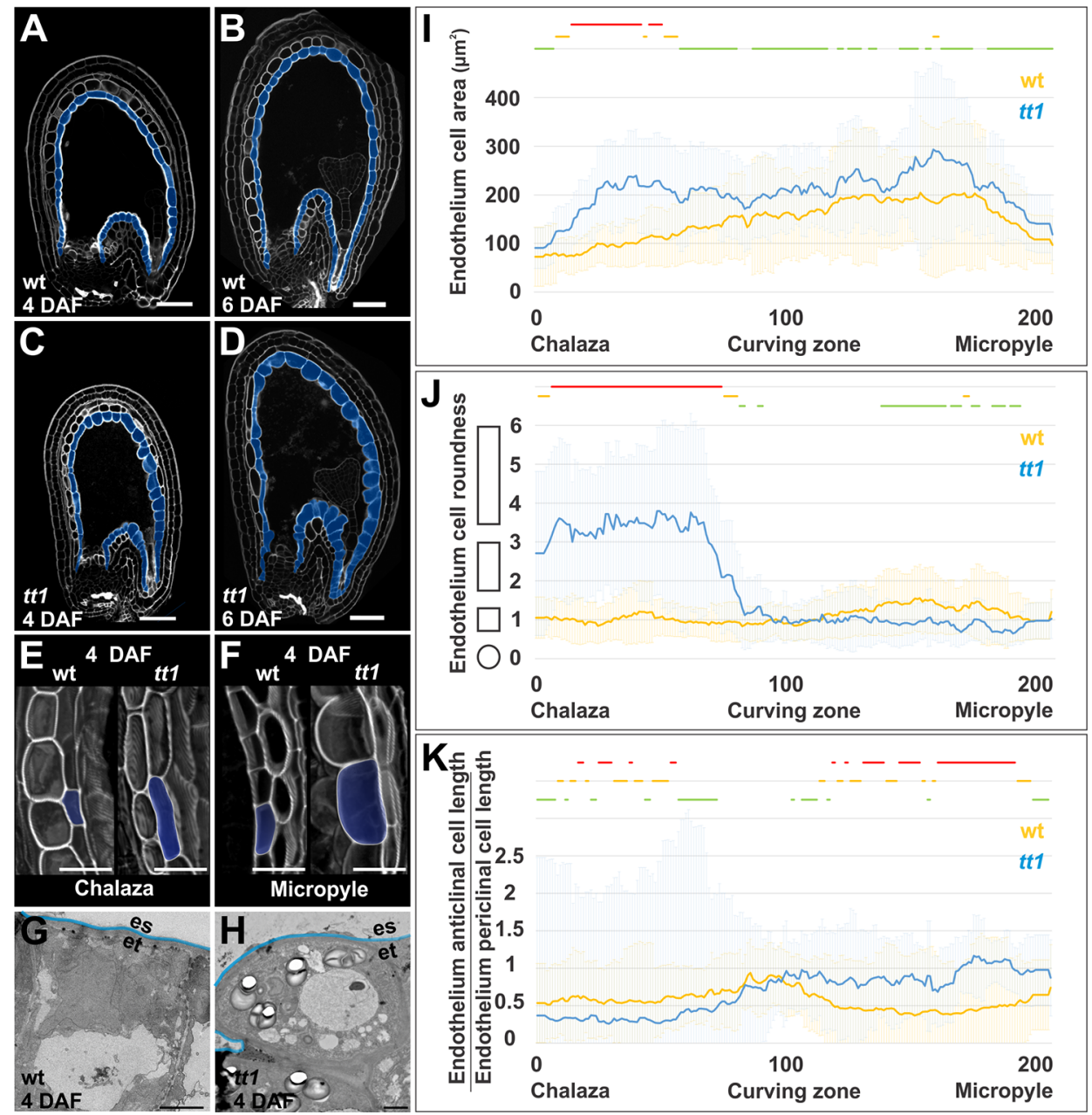

FIGURE 2 | TT1 regulates endothelium cell expansion. (A-D) Longitudinal mid-planes of wild type (wt) and tt1-4 seeds at 4 and 6 DAF, imaged using the mPS-PI technique. The endothelium is highlighted in blue. Ecotype Ws. Bars $=50 \mu \mathrm{m}$. (E, F) Longitudinal planes of three-dimensional reconstructed endothelium cells at the chalazal $(\mathbf{E})$ and micropylar $\mathbf{( F )}$ region of wild type (wt) and tt1-4 seeds at 4 DAF, imaged using the mPS-PI technique. One endothelium cell per sample is highlighted in blue. Ecotype Ws. Bars $=25 \mu \mathrm{m}$. (G, $\mathbf{H})$ Transmission electron microscopy images of wild type (wt) $(\mathbf{G})$ and $t t 1-3$ (H) endothelium cells at the micropylar region at 4 DAF. The interface between endosperm (es) and endothelium (en) is marked in blue. Ecotype Col-0. Bars = $25 \mu \mathrm{m}$. (I-K) Average endothelium cell area in $\left.\mu^{2} \mathbf{(} \mathbf{I}\right)$, endothelium cell roundness $\mathbf{( J )}$, and ratio between endothelium anticlinal and periclinal cell length $(\mathbf{K})$ (see Methods) along the seed coat proximal-distal axis (arbitrarily divided in 201 points) as observed in longitudinal mid-planes of wild type (wt, blue) and tt1-4 (yellow) seeds at 4 DAF. The shapes on the left of the graph in panel $\mathrm{J}$ exemplify how cell shape changes along the $y$-axis. Lines at the top of the graph indicate regions of statistically significant difference between wild type and tt1-4 (two-tailed Student's $t$-test; green line: $P<0.05$, orange line: $P<0.001$, red line: $P<0.00001$ ). Error bars indicate standard deviation (wild type $n=33, t t 1-4 n=20$ ). Ecotype Ws.

DAF, such $t t 1$ phenotypes were more pronounced (Figures 2B, D-F). To thoroughly characterize the effect of the $t t 1$ mutation on endothelium cell expansion, we quantified cell area and circularity along the endothelium proximal-distal axis in wild type and $t t 1$ seeds at 4 DAF. Overall, $t t 1$ endothelium cells exhibited an increased cell area when compared to the wild type (Figure 2I). Moreover, their shape appeared more elongated in the chalazal region and rounder in the micropylar region than in the wild type (Figure 2J). To examine the polarity of endothelium cell expansion, we calculated the ratio between anticlinal and periclinal cell length of wild type and $t t 1$ endothelium cells approximated to rectangles (see Methods).
Whereas the periclinal cell length was on average greater than the anticlinal cell length all along the wild type endothelium, $t t 1$ seeds exhibited anticlinally elongated cells in a sub-domain of the micropylar region (Figure $\mathbf{2 K}$ ).

It has been previously shown that longitudinal mid-planes of tt16 seeds displayed anticlinally over-elongated endothelium cells, as observed in the chalazal region of the $t t 1$ endothelium (Nesi et al., 2002; Coen et al., 2017). This $t$ t16 phenotype is due to the polarity of cell expansion more than to the extent of cell expansion (Coen et al., 2017). Similarly, three-dimensional images revealed that $t t 1$ chalazal endothelium cells were aligned along the proximal-distal axis of the seed, and thus 
perpendicular to wild-type cells (Figures 3A, B). Therefore, the phenotype of proximal $t t 1$ endothelium cells observed in two dimensional planes is due to cell orientation defects. By contrast, distal $t t 1$ endothelium cells appeared periclinally over-expanded, when compared to the wild type (Figures 3C, D). Altogether, these data indicate that TT1 modulates the polarity of cell expansion along the proximal-distal axis of the endothelium.

\section{TRANSPARENT TESTA 1 Regulates Inner Integument 1' Cell Expansion}

The iil' cell layer originates by periclinal cell divisions of the endothelium at the end of ovule development and undergoes severe cell expansion after fertilization (Schneitz et al., 1995; Debeaujon et al., 2003; Coen et al., 2017). To test if TT1 regulates iil' development, we studied iil' cell parameters in longitudinal mid-planes of wild type and $t t 1$ seeds at 4 and 6 DAF (Figures 4A-D). Quantitative analyses revealed that the shape of iil' cells at the chalazal region was significantly more elongated in $t t 1$ seeds than in wild type seeds, at 4 DAF (Figure 4E). Furthermore, some ii1' cells at the chalazal region appeared disconnected from the others in the $t t 1$ mutant, a phenotype never observed in the wild type (Figures 4A-D). To quantify this latter phenotype, we determined the ratio of total gap length to total length of the iil' chalazal region. Although high variability was observed, $t t 1$ mutant seeds at 4 DAF displayed on average
$4.6 \%$ of gaps along the length of the iil' chalazal region whereas all wild type seeds analyzed showed a continuous iil' cell layer (Figure 4F). Overall, this analysis indicates that TT1 regulates iil' cell expansion.

\section{TRANSPARENT TESTA 1 Promotes Endothelium and Inner Integument 1' Cell Division}

To test if the overall size of the ii is affected by the $t t 1$ mutation, we measured length and cell number of the gynobasal side of endothelium and iil' cell layers in seed longitudinal mid-planes. The ii1' cell layer of $t t 1$ seeds was shorter and displayed less cells than in the wild type, thus not compensating for reduced cell expansion (Figure 5A). By contrast, the $t$ t 1 endothelium appeared slightly longer and counted less cells, when compared to the wild type, thus partially compensating for cell orientation and over-expansion defects (Figure 5B). Overall, these data indicate that a size compensation mechanism is put in place only in the endothelium or that TT1 promotes cell division.

\section{TRANSPARENT TESTA 1 Is Stably Expressed in the Endothelium and Transiently in the Inner Integument 1'}

TT1 promoter analyses indicated that TT1 is expressed in the endothelium and, to a lesser extent, in the other integument cell
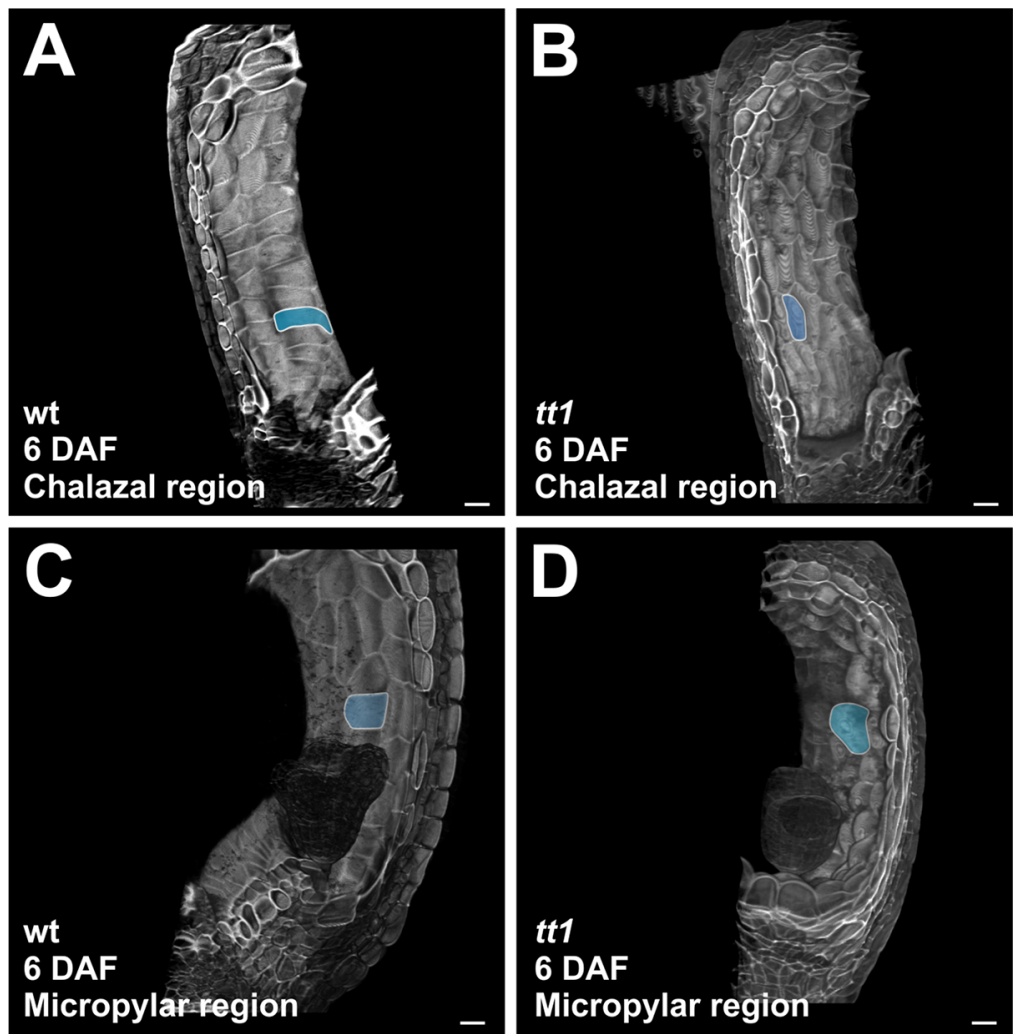

FIGURE 3 | П1 regulates the polarity of endothelium cell expansion. (A-D) Three-dimensionally reconstructed chalazal and micropylar regions of wild type (wt) and tt1-4 seeds at 6 DAF, imaged using the mPS-PI technique. One endothelium cell per sample is highlighted in blue. Ecotype Ws. Bars $=50 \mu m$. 

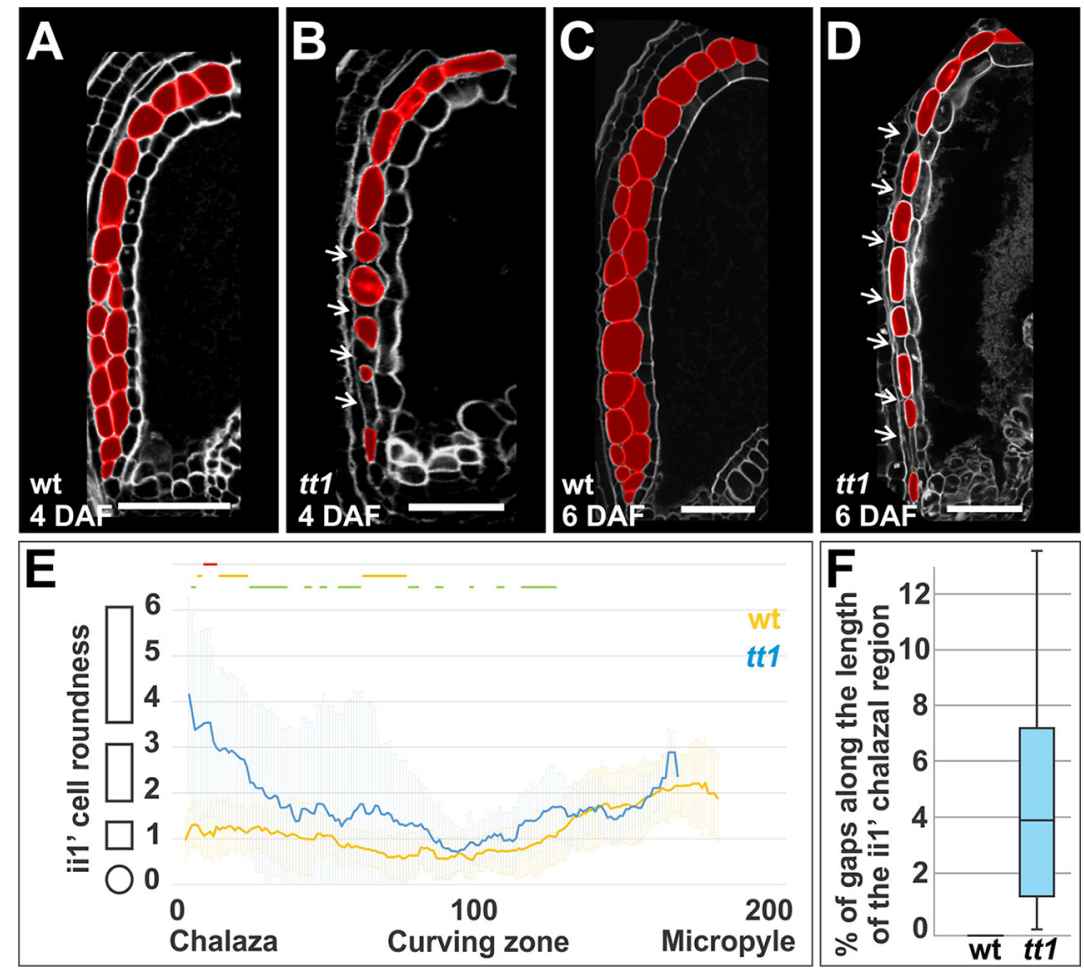

FIGURE 4 | TT1 regulates ii1' cell expansion. (A-D) Longitudinal mid-planes of wild type (wt) and tt1-4 seeds at 4 and 6 DAF, imaged using the mPS-PI technique. ii1' and ii1"' cells are highlighted in red. Ecotype Ws. Bars $=50 \mu \mathrm{m}$. (E) Average ii1' cell roundness (see Methods) along the seed coat proximal-distal axis (arbitrarily divided in 201 points) as observed in longitudinal mid-planes of wild type (wt, blue) and tt1-4 (yellow) seeds at 4 DAF. The shapes on the left of the graph exemplify how cell shape changes along the $y$-axis. Lines at the top of the graph indicate regions of statistically significant difference between wild type and $t t 1$ - 4 (two-tailed Student's $t$-test; green line: $P<0.05$, orange line: $P<0.001$, red line: $P<0.00001)$. Error bars indicate standard deviation (wild type $n=33$, $t t 1-4 n=20)$. Ecotype Ws. (F) Box plot representing the percentage of gaps along the length of the ii1' chalazal region in wild type (wt) and $t t 1-4$ seeds 4 DAF. (wild type $n=33$, $t t 1-4 n=$ 20). Ecotype Ws.

layers (; ). RNA in situ hybridization experiments showed TT1 expression in the endothelium but high background signal levels did not allow to conclusively address TT1 expression in other integument cell layers (). The promoter region of CmWIP1, TT1 orthologue in melon, contains retrotransposons whose methylation regulates CmWIP1 expression (Coen et al., 2019). Likewise, the promoter region of TT1 is annotated as carrying copia-like retrotransposons and $\mathrm{RC} / \mathrm{Helitron}$ and DNA/MuDR transposon fragments, thus suggesting that its expression might also be regulated by cis-epigenetic mechanisms. Since previous studies included a relatively small TT1 promoter sequence downstream of the transposon region and to account for the possibility of TT1 post-translational regulation, we created a marker line carrying TT1 $3 \mathrm{~kb}$ promoter region (including part of the transposon region) upstream of TT1 genomic sequence translationally fused to GFP (3kbProTT1:gTT1-GFP). Seven independent 3kbProTT1:gTT1-GFP lines showed fluorescence in the nuclei of endothelium cells from stage 2-IV of ovule development (Schneitz et al., 1995) till globular embryo stage of seed development (Figures 6A-F). In newly periclinally divided endothelium cells we observed fluorescence both in endothelium and iil' cells (Figure 6C). Furthermore, we detected GFP fluorescence in the most proximal iil' cells (Figures 6B-F) and in a subdomain of the seed chalaza (Figure 6D). Non-nuclear auto-fluorescence signal is also visible in the seed coat (Figures 6B-E). Overall, these data confirm TT1 expression in the endothelium and suggest a novel layer of transcriptional and/ or post-transcriptional regulation in the other integument cells layers and in the chalaza, when compared to previously published data (Sagasser et al., 2002; Coen et al., 2019). Finally, we did not detect any significant change in TT1 expression across fertilization by RT-qPCR analyses (Figure 6G).

\section{TRANSPARENT TESTA 1 Acts Downstream of TRANSPARENT TESTA 16 At the Chalazal Region}

The MADS box transcription factor genes TT16, STK, SHP1, and SHP2 are known to affect the development of the ii (Nesi et al., 2002; Mizzotti et al., 2012; Mizzotti et al., 2014; Ehlers et al., 2016; Coen et al., 2017). To determine their genetic interactions with TT1, we tested their expression levels in each other mutant background relative to wild type. Whereas TT16, SHP1, and SHP2 expression was not affected by the $t t 1$ mutation, STK expression was mildly downregulated in $t t 1$ seeds at $4 \mathrm{DAF}$, when compared to the wild type (Figure 7A). Conversely, TT1 


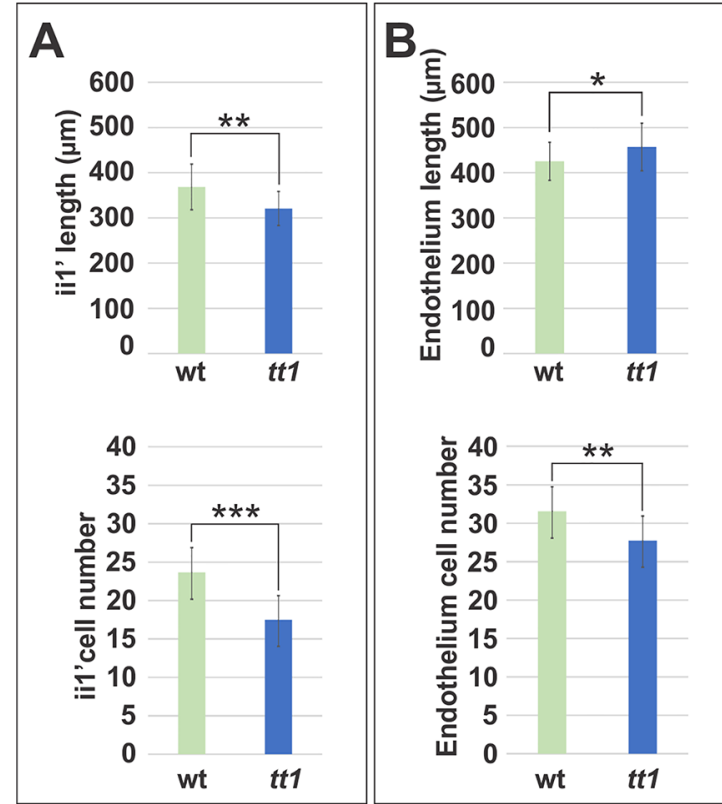

FIGURE 5 | T1 regulates ii size. (A, B) Average length and cell number of the gynobasal side of ii1' (A) and endothelium (B) cell layers in seed longitudinal mid-planes at 4 DAF. Asterisks indicate statistical significance between wild type and tt1-4 (two-tailed Student's $t$-test; *: $\mathrm{P}<0.05$, ${ }^{\star *}$ : $\mathrm{P}<$ $0.001,{ }^{\star \star \star}: \mathrm{P}<0.00001$ ). Error bars indicate standard deviation (wild type $\mathrm{n}=$ 33, $t t 1-4 n=20)$. Ecotype Ws.
mRNA levels were unchanged in stk and shp1;shp2 mutants while drastically down-regulated in $t t 16$ seeds at $4 \mathrm{DAF}$, when compared to the wild type (Figure 7A).

TT16 has been shown to have fertilization-dependent functions, such as nucellus elimination and PA deposition (Nesi et al., 2002; Xu et al., 2016). To test if TT16 regulates TT1 expression in a fertilization-dependent manner, we analyzed wild type and $t$ t16 ovules (0 DAF) and seeds (2 DAF). TT1 expression was equally reduced before and after fertilization in the $t t 16$ mutant when compared to the wild type (Figure 7A).

To thoroughly characterize TT16 role in regulating TT1 expression, we introgressed the $1 \mathrm{kbProTT1}$ NTF marker line (Coen et al., 2019), coding for a GFP chimeric protein that binds to the nuclear membrane (Deal and Henikoff, 2011) under the control of the TT1 $1 \mathrm{~kb}$ promoter region, in a tt16 mutant background. Whereas we detected GFP fluorescence all along the endothelium of $1 \mathrm{kbProTT1}$ : NTF seeds (Figures 7B, C), the GFP signal was absent in the chalazal region of the endothelium of $1 \mathrm{kbProTT1}$ :NTF; tt16 seeds (Figures 7D, E). Non-nuclear auto-fluorescence signal is also visible in the seed coat (Figures 7B-E and Supplemental Figure 2). Altogether, these results suggest that TT16 positively regulates TT1 expression solely in chalazal endothelium cells.

In line with our transcriptional analyses, longitudinal midplanes of $t t 1$ and $t t 16$ seeds displayed equally over-elongated endothelium cells at the chalazal region when compared to the wild type (Figures 7F-H). To further characterize TT1 and TT16 genetic interaction, we created a $t t 1 ; t t 16$ double mutant. The chalazal region of the $t t 1 ; t t 16$ inner integument displayed over-
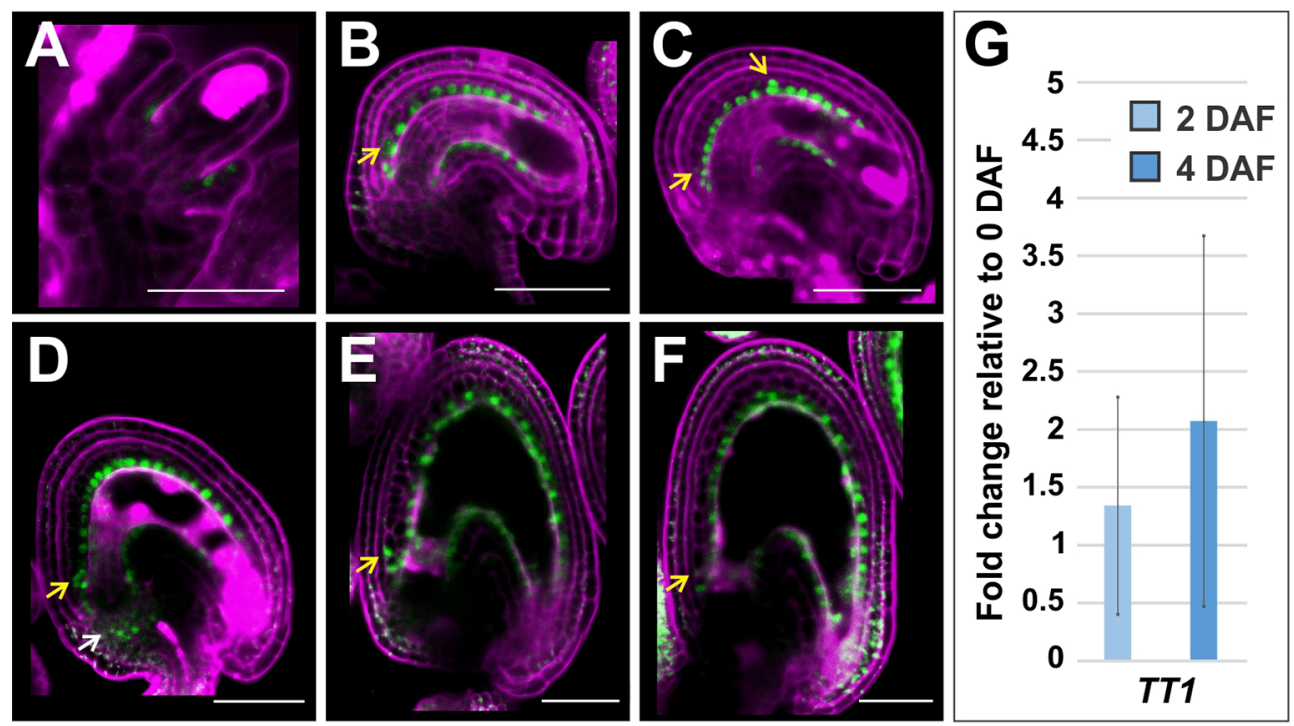

FIGURE 6 | TT1 expression in ovules and seeds. (A-F) GFP fluorescence images of 3kbProTT1:gTT1-GFP ovules at stage 2-V (A), stage 3-VI (B), and maturity (C) and seeds at 1 DAF (D), 2 DAF (E), and 3 DAF (F). Yellow and white arrows point to GFP fluorescent ii1' and chalazal cells, respectively. GFP and propidium iodide fluorescence are in green and purple, respectively. Ecotype Col-0. Bars $=50 \mu \mathrm{m}$. (G) Quantitative RT-PCR analysis of $\pi 1$ expression in wild type seeds at 2 and 4 DAF versus seeds at 0 DAF. Error bars indicate standard deviation. Expression levels were normalized and averaged from four independent biological samples. Ecotype Col-0. 

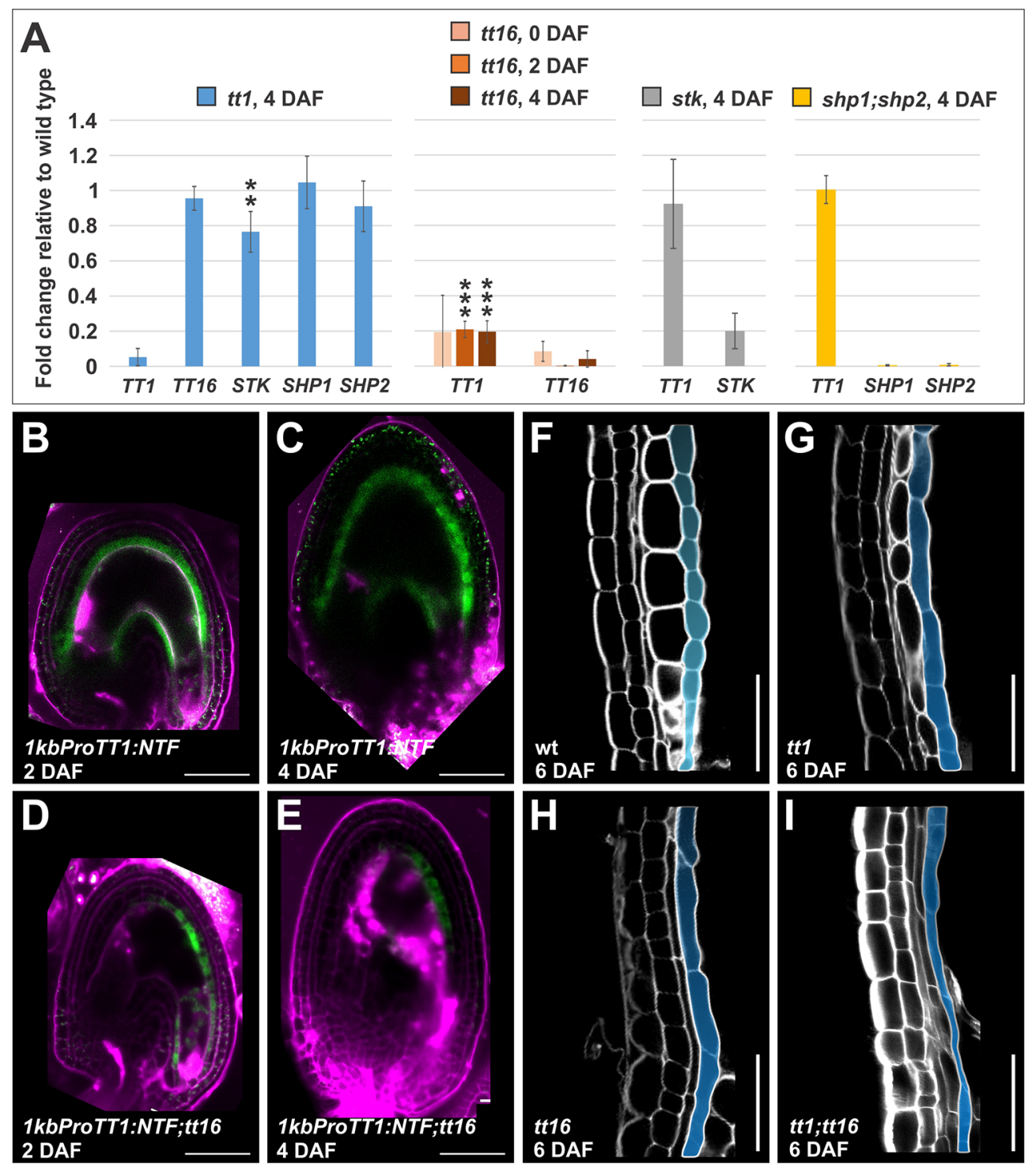

FIGURE 7 | $\Pi 116$ regulates $\pi 1$ expression. (A) Quantitative RT-PCR analyses of $\pi 1$, $\Pi 116$, STK, SHP1, and SHP2 expression in tt1-3, tt16-1, stk-2, and shp1-1; shp2-1 mutant seeds relative to wild type. Asterisks indicate statistical significance between wild type and mutant (two-tailed Student's $t$-test; ${ }^{\star \star}$ : P < 0.001 , ${ }^{\star \star *}: \mathrm{P}<$ 0.00001). Error bars indicate standard deviation. Expression levels were normalized and averaged from four independent biological samples. Ecotype Col-0. (B-E) GFP fluorescence images of 1kbProTT1:NTF (C, D) and 1kbProTT1:NTF;tt16-1 (E, F) seeds at 2 (C, E) and 4 (D, F) DAF. GFP and propidium iodide fluorescence are in green and purple, respectively. Ecotype Col-0. Bars $=50 \mu \mathrm{m}$. (F-l) Longitudinal mid-planes of integument cells at the chalazal area of wild type (wt) (G), tt1-4 (H), tt16-1 (I), and tt1-4;tt16-1 (J) seeds at 6 DAF, imaged using the mPS-PI technique. Endothelium cells are highlighted in blue. Ecotype Ws. Bars $=50 \mu m$.

elongated endothelium cells as observed in either single mutant line (Figure 7G-I). Overall, these data indicate that TT1 is epistatic to TT16 in endothelium cell development at the chalazal region.

\section{TRANSPARENT TESTA 1 Downstream Target Genes}

Our analysis of TT1 expression pattern and loss of function mutant phenotypes suggest a role for TT1 in establishing endothelium cell identity. The endothelium is an epidermal cell layer (Schneitz et al., 1995) and expresses the MERISTEM
LAYER 1 (ML1) (Supplemental Figure 3), PROTODERMAL FACTOR 2 (PDF2), and CRINKLY4 (CR4) epidermal cell fate genes (Lu et al., 1996; Sessions et al., 1999; Abe et al., 2003; Gifford et al., 2003; San-Bento et al., 2014; Huang et al., 2016). Nevertheless, ML1, PDF2, and CR4 expression was not affected by the $t t 1$ mutation, when compared to the wild type (Figure 8A).

The TT1 gene has been first identified for its loss of function transparent TESTA phenotype (Sagasser et al., 2002) and has been shown to positively regulate PA biosynthetic genes (Appelhagen et al., 2011). To further characterize its role in PA biosynthesis, we 

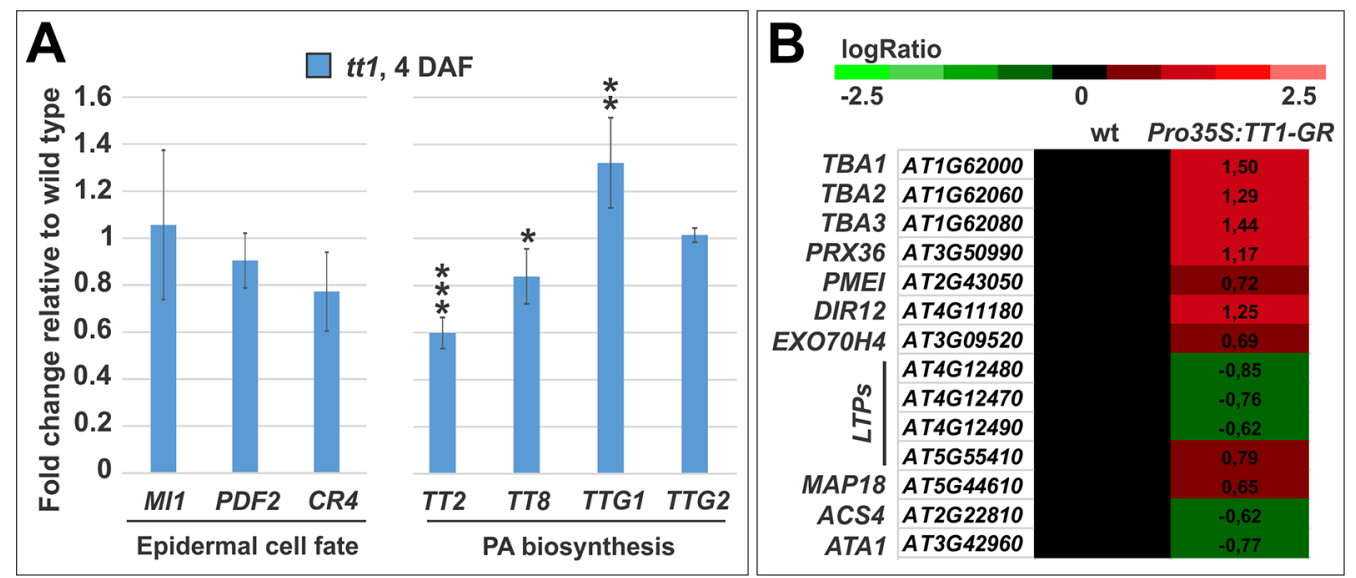

FIGURE 8 | T11 downstream target genes (A) Quantitative RT-PCR analyses of ML1, PDF2, CR4, TT2, TT, TTG1, and TTG2 expression in tt1-3 mutant seeds relative to wild type. Asterisks indicate statistical significance between wild type and mutant (two-tailed Student's $t$-test; ${ }^{*}$ : $P<0.05,{ }^{\star \star}$ : $P<0.001,{ }^{\star \star \star}$ : $\mathrm{P}<0.00001$ ). Error bars indicate standard deviation. Expression levels were normalized and averaged from four independent biological samples. Ecotype Col-0. (B) Fold change values in gene expression between mock and DEX treated siliques in wild type (wt) and 35S:TT1-GR backgrounds as detected by microarray analyses. Ecotype Col-0.

tested the expression of key regulatory genes of the PA pathway in the tt1 mutant. TT2, TT8, TRANSPARENT TESTA GLABRA 1 (TTG1), and TTG2 encode for transcription factors that regulate the flavonoid late metabolism in developing siliques. TT2 and TT8 were down-regulated whereas TTG1 up-regulated in $t t 1$ seeds at $4 \mathrm{DAF}$, when compared to the wild type (Figure 8A).

To identify novel TT1 downstream target genes, we created an inducible TT1 transcription factor fused to the rat glucocorticoid receptor (GR) under the control of the constitutive cauliflower mosaic virus $35 \mathrm{~S}$ promoter (Pro35S: TT1-GR). Dexamethasone (DEX) treatment releases the GR transcription factor chimeric protein from a cytoplasmic HEAT SHOCK PROTEIN 90 complex that prevents its nuclear translocation and therefore its functionality (Schena et al., 1991). Addition of DEX to Pro35S:TT1-GR plants caused leaf elongation and narrowing similar to plants that overexpress TT1 (Sagasser 2002), indicating that the TT1-GR chimeric protein retains function. To minimize sample handling, we analyzed entire inflorescences bearing ovules and seeds up to the early torpedo embryo stage. To prevent indirect transcriptional effects of the inducible TT1-GR protein, we infiltrated Pro35S:TT1-GR inflorescences with cycloheximide (CHX), an inhibitor of protein synthesis. We then treated the samples with DEX or a mock solution for $1 \mathrm{~h}$. To discount for the unspecific effect of DEX, we conducted an identical experiment with wild type inflorescences. Transcript levels across the entire genome were measured by hybridization to CATMA microarrays (Allemeersch et al., 2005). To increase our ability to find genuine targets (i.e., decrease false negatives) and reduce the background of false positives, we concentrated on genes that showed a statistically significant interaction between time of treatment and genotype. This approach identified 74 up-regulated and 16 down-regulated genes by DEX induction in the Pro35S:TT1-GR line, which were unaffected in the wild type background (Supplemental Table 1). To investigate the range of processes that could be regulated by TT1, we performed a GO annotation analysis. We detected response to stress and stimulus, developmental processes, protein metabolism, cell organization and biogenesis, signal transduction, transport, and transcription DNA-dependent categories.

A GO enrichment analysis for biological processes revealed significant enrichments in GO annotations in mucilage biosynthetic process and seed coat development. Among the proteins regulated by TT1-GR, we detected a known mucilage extrusion factor, PEROXIDASE 36 (PRX36) (Kunieda et al., 2013), the DIRIGENT 12 (DIR12) protein, which is a member of the PRX36 co-expression network (Ranocha et al., 2014), three abundant mucilage proteins, TESTA ABUNDANT 1 (TBA1), TBA2, and TBA3 (Tsai et al., 2017), a mucilage related (Voiniciuc et al., 2015) PECTIN METHYL ESTERASE INHIBITOR (PMEI), which promotes mucilage release (Saez-Aguayo et al., 2013), and the EXOCYST SUBUNIT EXO70 FAMILY PROTEIN H4 (EXO70H4), a subunit of the exocyst complex that participates in mucilage deposition (Kulich et al., 2010) (Figure 8B).

A GO enrichment analysis for cellular components showed an enrichment in apoplastic proteins. Among the genes annotated to be secreted, we found that TT1-GR regulates the expression of four LIPID TRANSFER PROTEIN (LTP) genes (Figure 8B). LTPs have been implicated in cuticle formation and cell wall extension (Salminen et al., 2016), which are both processes regulated by TT1 (Loubery et al., 2018; Coen et al., 2019).

Validation of our microarray analysis comes from the finding of the only experimentally verified TT1 downstream target gene. CmWIP1, TT1 orthologue in melon, has been shown to repress the expression of the melon 1-AMINOCYCLOPROPANE-1CARBOXYLIC ACID SYNTHASE 7 (ACS7), an ethylene biosynthetic enzyme that regulates sex determination. In line with this study, we revealed that TT1-GR represses ACS4, an orthologue of $A C S 7$, and regulates the expression of four ethylene responsive factor genes (Figure 8B and Supplemental Table 1). Furthermore, TT1 downregulated the expression of 
ARABIDOPSIS TAPETUM 1 (ATA1), the orthologue of the TASSLE SEED 2 gene that controls sex determination in maize (DeLong et al., 1993) (Figure 8B). By contrast, we did not detect PA regulatory or biosynthetic genes.

Finally, to find candidate genes possibly responsible for the cell expansion phenotype of $t t 1$ endothelium cells, we looked for target genes annotated as cell shape regulators. This screen retrieved only the MICROTUBULE-ASSOCIATED PROTEIN 18 (MAP18) (also known as PLASMA MEMBRANE ASSOCIATED CA2+-BINDING PROTEIN-2, PCaP2), which regulates directional cell growth and cortical microtubule organization (Figure 8B) (Wang et al., 2007; Kato et al., 2019). According to the laser microdissection transcriptomics data by Le and coworkers (Le et al., 2010), MAP18 is expressed in the seed coat at the linear cotyledon stage. Nevertheless, map18 mutant seeds displayed wild type looking endothelium cells (Supplemental Figure 1).

\section{DISCUSSION}

The seed coat, as a whole, is a highly polar structure. Nevertheless, less is known about the development of polar axes in individual integument cell layers. Here, we characterize the role of the Arabidopsis TT1 transcription factor in modulating polarity along the proximal-distal axis of the endothelium and shed light on TT1 upstream and downstream regulatory pathways.

\section{TRANSPARENT TESTA 1 and TRANSPARENT TESTA 16 Modulate Cell Expansion Along the Endothelium Proximal-Distal Polarity Axis}

We identified proximal-distal polarity along the wild type endothelium and showed that it is severely affected by the $t t 1$ mutation. At the chalazal region, $t t 1$ endothelium cells appeared oriented along the proximal-distal axis, perpendicularly to wild type cells. By contrast, $t t 1$ endothelium cells at the micropylar region were more periclinally expanded on their adaxial side. These data indicate that TT1 regulates both orientation and extent of cell expansion (Figure 9). Morphological defects of $t t 1$ seeds as well as TT1 early expression in ovules favor the hypothesis that TT1 regulates endothelium development and only indirectly PA biosynthesis. In line with this hypothesis, we detected lower expression of PA regulatory genes in $t t 1$ seeds, possibly responsible for the down regulation of PA biosynthetic genes previously described in $t t 1$ seeds (Appelhagen et al., 2011), but we did not find either category of genes in transcriptomic analyses aimed at revealing TT1 immediate target genes. Alternatively, PA biosynthetic genes might be regulated by TT1-TT2 protein complexes, as suggested by Appelhagen and coworkers (Appelhagen et al., 2011), thus requiring the induction of both transcription factors to affect PA biosynthesis.

We discovered that TT16 promotes TT1 expression in the chalazal but not micropylar region of the endothelium. In agreement with this result, $t t 16$ and $t t 1$ seeds show the same

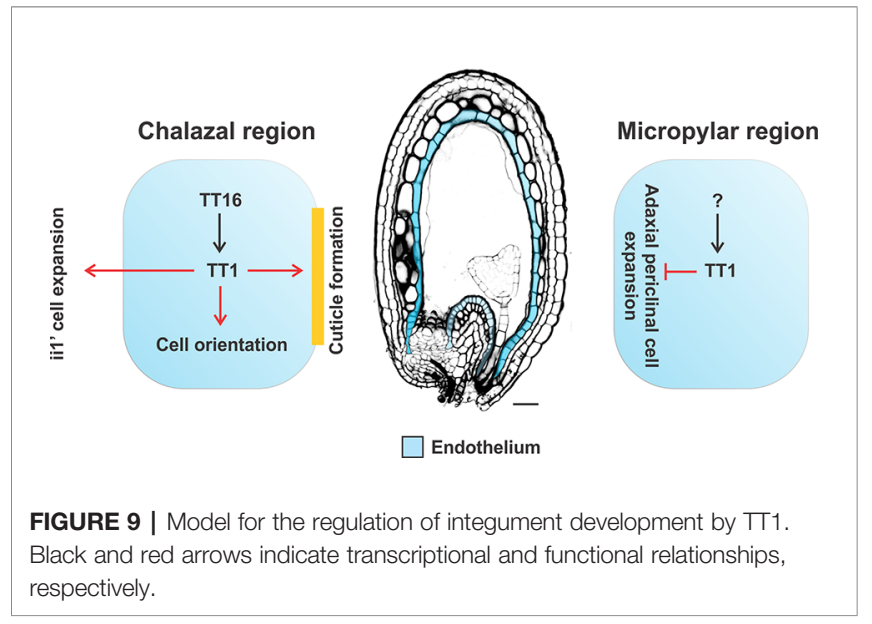

lack of cuticle and cell orientation phenotypes in the chalazal endothelium whereas opposite phenotypes in the micropylar region (Figure 9) (Nesi et al., 2002; Coen et al., 2017; Coen et al., 2019). We hypothesize that a different transcriptional regulator might control TT1 expression in the micropylar region of the endothelium (Figure 9). Downstream of TT1, we discovered MAP18 as putative target gene responsible for endothelium cell polar growth. MAP18 has been shown to contribute to directional cell growth and cortical microtubule organization (Wang et al., 2007; Kato et al., 2019). Nevertheless, map18 seeds did not display any obvious mutant phenotype possibly because of gene redundancy. Furthermore, TT1 affected the expression of a number of genes involved in the ethylene pathway, as previously shown in melon (Martin et al., 2009). Ethylene regulates anisotropic cell growth and might therefore be responsible for $t t 1$ cell expansion phenotypes (Kieber et al., 1993; Rodrigues-Pousada et al., 1993; Xu et al., 2008). Finally, our microarray data revealed LTP genes as putative TT1 downstream targets. The tobacco NtLTP1.6 has been found to regulate cell wall extension, thus suggesting that LTPs might affect endothelium cell elongation. LTPs play also a role in intracuticular or epicuticular wax deposition (Cameron et al., 2006). The adaxial side of the endothelium is covered by a cuticle layer that lacks or is low in waxes (Coen et al., 2019). TT1, which has been shown to promote cuticle deposition (Loubery et al., 2018; Coen et al., 2019), might therefore prevent wax accumulation by repressing LTPs expression. The cuticle creates a mechanical barrier between the endothelium and the endosperm that might repress cell expansion and be partly responsible for the flat adaxial surface of wild type endothelium cells. Nevertheless, $t t 16$ endothelium cells, which display a more dramatic cuticle phenotype than $t t 1$, do not expand periclinally as in $t t 1$ seeds, indicating that the cuticle alone is not enough to explain the squared shape of wild type endothelium cells (Coen et al., 2017; Coen et al., 2019).

We hypothesize that changes in cell size, shape, and orientation along the endothelium proximal-distal axis might be necessary to achieve proper seed shape. In line with this interpretation, the chalazal region of $t t 1$ seeds is flatter than that 
of wild type seeds, a phenotype also observed in $t$ t16 seeds (Nesi et al., 2002). Furthermore, reduced periclinal cell expansion at the endothelium micropylar region might be important to create an empty groove that allows the correct growth of the embryo. In the $t 16$ mutant, the iil' cell layer extends toward the micropyle, thus narrowing the micropylar region and preventing, in some extreme cases, embryo growth (Coen et al., 2017). Similarly, TT1 might prevent the expansion of micropylar endothelium cells to facilitate embryo development. On the other hand, the seeds of a number of plant species, whose micropylar end is formed by the ii and not the oi as in Arabidopsis, show endothelium cell thickening as a way to restrict the micropylar pore (Coen and Magnani, 2018). Further evidence for the importance of an endothelium proximal-distal polarity axis comes from the lack of cuticle at the seed micropylar end, which has been suggested to allow diffusion of nutrients and developmental signals from zygotic to maternal tissues (Loubery et al., 2018; Coen et al., 2019). We, therefore, speculate that regulation of TT1 function or expression might be responsible for such natural morphological diversity.

\section{TRANSPARENT TESTA 1 Promotes Inner Integument 1' Cell Expansion and Fertilization Responsiveness}

The ii' cell layer is the only integument cell layer that does not respond to the endosperm signal that relieves the repressive action of the FERTILIZATION INDEPENDENT ENDOSPERM (FIE) and MULTICOPY SUPPRESSOR OF IRA1 (MSI1) Polycomb Group (PcG) proteins, which prevent the fertilizationindependent expansion of the integuments (Roszak and Kohler, 2011). A fraction of unfertilized fie/+ and msil/+ ovules develop into enlarged autonomous seeds that exhibit a developed seed coat, which accumulates PAs, and a degenerated nucellus, both hallmarks of fertilization (Roszak and Kohler, 2011; Xu et al., 2016). However, fie/+ and msil/t enlarged autonomous seeds display an underdeveloped and discontinuous iil' cell layer made of unexpanded cells and empty spaces (Coen et al., 2017; Fiume et al., 2017b), as observed in $t t 1$ seeds. These data suggest that TT1 promote FIE- and MSI1-independent expansion of iil' cells.

The iil' cell layer differs from the other cell layers for its subepidermal position and origin by periclinal cell divisions. It has been previously shown that sub-epidermal outer integument cell stripes, developing in a fraction of wild type seeds, respond to the FIE and MSI1 repressive mechanism as epidermal integument cell layers (Fiume et al., 2017a). This study suggests that the subepidermal position of the iil' is not sufficient to explain for its insensitivity to FIE and MSIl and points to an asymmetric periclinal cell division of the endothelium as a more likely scenario. Our transcriptional analysis showed a more restricted and transient expression of TT1 in the iil' cell layer than previously described (Sagasser et al., 2002; Coen et al., 2019). We detected TT1 expression solely in newly developed iil' cells, right after endothelium periclinal cell division, and in the most proximal ii1' cells. Compared to previous analyses, we tested a longer promoter region containing transposon sequences, which have been shown to regulate TT1 orthologue expression in melon (Martin et al., 2009), and TT1 genomic sequence. These data suggest that Arabidopsis TT1 expression in the iil' might be negatively regulated by transposons or TT1 intronic sequence. In an alternative non-exclusive scenario, TT1 protein might be rapidly degraded in the iil' cell layer. TT1 protein pattern across fertilization, constantly present in the endothelium while only transiently in most iil' cells, might explain for the iil' unique responsiveness to fertilization.

Our data show that TT1 expression in the iil' is activated by TT16. It has been shown that TT16 regulates iil' cell expansion and patterning along the proximal-distal axis (Mizzotti et al., 2012; Coen et al., 2017). In line with these results, longitudinal mid-planes of both tt1 and tt16 seeds display iil' cells with an anticlinally over-elongated shape when compared to the wild-type (Coen et al., 2017). Nevertheless, $t t 16$ seeds do not display empty spaces in between iil' cells, thus suggesting that TT1 and TT16 also play independent functions as observed in the endothelium micropylar region.

\section{DATA AVAILABILITY STATEMENT}

The datasets generated for this study can be found in the Microarray data from this article were deposited in the international repository GEO, Gene Expression Omnibus (Edgar et al., 2002), accession number: GSE 134014. All steps of the experiment, from growth conditions to bioinformatic and statistical analyses, were detailed in CATdb (Gagnot et al., 2008) (Project: RA15-05_TT1) according to the "Minimum Information About a Microarray Experiment" standards.

\section{AUTHOR CONTRIBUTIONS}

OC performed the research, analyzed the data, and helped to write the article. JL, WX, and DG helped to perform morphological analyses, SP performed the transcriptomic analysis and CP performed the transmission electron microscopy analysis. LL helped to analyze the data and write the article. EM designed the research and wrote the article.

\section{ACKNOWLEDGMENTS}

We thank Adrienne H.K. Roeder for the ProML1:gML1-mCitrine; ml1-3 line, Annette Becker for the shp1;shp2 mutant, Helen North for helpful discussions and the Observatoire du Végétal for plant culture, access to imaging facilities and assistance. The IJPB and IPS2 institutes benefit from the support of the Labex Saclay Plant Sciences-SPS (ANR-10-LABX-0040-SPS).

\section{SUPPLEMENTARY MATERIAL}

The Supplementary Material for this article can be found online at: https://www.frontiersin.org/articles/10.3389/fpls.2019.01801/ full\#supplementary-material 


\section{REFERENCES}

Abe, M., Katsumata, H., Komeda, Y., and Takahashi, T. (2003). Regulation of shoot epidermal cell differentiation by a pair of homeodomain proteins in Arabidopsis. Development 130 (4), 635-643. doi: 10.1242/dev.00292

Allemeersch, J., Durinck, S., Vanderhaeghen, R., Alard, P., Maes, R., Seeuws, K., et al. (2005). Benchmarking the CATMA microarray. A novel tool for Arabidopsis transcriptome analysis. Plant Physiol. 137 (2), 588-601. doi: $10.1104 /$ pp. 104.051300

Appelhagen, I., Lu, G. H., Huep, G., Schmelzer, E., Weisshaar, B., and Sagasser, M. (2011). TRANSPARENT TESTA1 interacts with R2R3-MYB factors and affects early and late steps of flavonoid biosynthesis in the endothelium of Arabidopsis thaliana seeds. Plant J. 67 (3), 406-419. doi: 10.1111/j.1365313X.2011.04603.x

Baudry, A., Heim, M. A., Dubreucq, B., Caboche, M., Weisshaar, B., and Lepiniec, L. (2004). TT2, TT8, and TTG1 synergistically specify the expression of BANYULS and proanthocyanidin biosynthesis in Arabidopsis thaliana. Plant J. 39 (3), 366-380. doi: 10.1111/j.1365-313X.2004.02138.x

Boyes, D. C., Zayed, A. M., Ascenzi, R., McCaskill, A. J., Hoffman, N. E., Davis, K. R., et al. (2001). Growth stage-based phenotypic analysis of Arabidopsis: a model for high throughput functional genomics in plants. Plant Cell 13 (7), 1499-1510. doi: 10.2307/3871382

Brunaud, V., Balzergue, S., Dubreucq, B., Aubourg, S., Samson, F., Chauvin, S., et al. (2002). T-DNA integration into the Arabidopsis genome depends on sequences of pre-insertion sites. EMBO Rep. 3 (12), 1152-1157. doi: 10.1093/ embo-reports/kvf237

Cameron, K. D., Teece, M. A., and Smart, L. B. (2006). Increased accumulation of cuticular wax and expression of lipid transfer protein in response to periodic drying events in leaves of tree tobacco. Plant Physiol. 140 (1), 176-183. doi: 10.1104/pp.105.069724

Clough, S. J., and Bent, A. F. (1998). Floral dip: a simplified method for Agrobacterium-mediated transformation of Arabidopsis thaliana. Plant J. 16 (6), 735-743. doi: 10.1046/j.1365-313x.1998.00343.x

Coen, O., and Magnani, E. (2018). Seed coat thickness in the evolution of angiosperms. Cell Mol. Life Sci. 75 (14), 2509-2518. doi: 10.1007/s00018018-2816-x

Coen, O., Fiume, E., Xu, W., De Vos, D., Lu, J., Pechoux, C., et al. (2017). Developmental patterning of the sub-epidermal integument cell layer in Arabidopsis seeds. Development 144 (8), 1490-1497. doi: 10.1242/dev.146274

Coen, O., Lu, J., Xu, W., De Vos, D., Pechoux, C., Domergue, F., et al. (2019). Deposition of a cutin apoplastic barrier separating seed maternal and zygotic tissues. BMC Plant Biol. 19 (1), 304. doi: 10.1186/s12870-019-1877-9

De Giorgi, J., Piskurewicz, U., Loubery, S., Utz-Pugin, A., Bailly, C., Mene-Saffrane, L., et al. (2015). An endosperm-associated cuticle is required for arabidopsis seed viability, dormancy and early control of germination. PloS Genet. 11 (12), e1005708. doi: 10.1371/journal.pgen.1005708

Deal, R. B., and Henikoff, S. (2011). The INTACT method for cell type-specific gene expression and chromatin profiling in Arabidopsis thaliana. Nat. Protoc. 6 (1), 56-68. doi: 10.1038/nprot.2010.175

Debeaujon, I., Nesi, N., Perez, P., Devic, M., Grandjean, O., Caboche, M., et al. (2003). Proanthocyanidin-accumulating cells in Arabidopsis testa: regulation of differentiation and role in seed development. Plant Cell 15 (11), 2514-2531. doi: $10.1105 /$ tpc. 014043

Dekkers, B. J., Willems, L., Bassel, G. W., van Bolderen-Veldkamp, R. P., Ligterink, W., Hilhorst, H. W., et al. (2012). Identification of reference genes for RTqPCR expression analysis in Arabidopsis and tomato seeds. Plant Cell Physiol. 53 (1), 28-37. doi: 10.1093/pcp/pcr113

DeLong, A., Calderon-Urrea, A., and Dellaporta, S. L. (1993). Sex determination gene TASSELSEED2 of maize encodes a short-chain alcohol dehydrogenase required for stage-specific floral organ abortion. Cell 74 (4), 757-768. doi: 0092-8674(93)90522-R doi: 10.1016/0092-8674(93)90522-R

Eden, E., Navon, R., Steinfeld, I., Lipson, D., and Yakhini, Z. (2009). GOrilla: a tool for discovery and visualization of enriched GO terms in ranked gene lists. $B M C$ Bioinf. 10, 48. doi: 10.1186/1471-2105-10-48

Edgar, R., Domrachev, M., and Lash, A. E. (2002). Gene Expression Omnibus: NCBI gene expression and hybridization array data repository. Nucleic Acids Res. 30 (1), 207-210. doi: 10.1093/nar/30.1.207
Ehlers, K., Bhide, A. S., Tekleyohans, D. G., Wittkop, B., Snowdon, R. J., and Becker, A. (2016). The MADS Box Genes ABS, SHP1, and SHP2 are essential for the coordination of cell divisions in ovule and seed coat development and for endosperm formation in arabidopsis thaliana. PloS One 11 (10), e0165075. PONE-D-16-29187 [pii] doi: 10.1371/journal.pone.0165075

Figueiredo, D. D., Batista, R. A., Roszak, P. J., Hennig, L., and Kohler, C. (2016). Auxin production in the endosperm drives seed coat development in Arabidopsis. Elife 5 doi: 10.7554/eLife.20542

Fiume, E., Coen, O., Xu, W., Lepiniec, L., and Magnani, E. (2017a) Developmental patterning of sub-epidermal cells in the outer integument of Arabidopsis seeds. PloS One 12 (11), e0188148. PONE-D-17-29628[pii] doi: 10.1371/ journal.pone. 0188148

Fiume, E., Coen, O., Xu, W., Lepiniec, L., and Magnani, E. (2017b). Growth of the Arabidopsis sub-epidermal integument cell layers might require an endosperm signal. Plant Signal Behav. 12 (8), e1339000. doi: 10.1080/ 15592324.2017.1339000

Gagnot, S., Tamby, J. P., Martin-Magniette, M. L., Bitton, F., Taconnat, L., Balzergue, S., et al. (2008). ). CATdb: a public access to Arabidopsis transcriptome data from the URGV-CATMA platform. Nucleic Acids Res. 36 ((Database issue)), D986-D990. doi: 10.1093/nar/gkm757

Garcia, D., Fitz Gerald, J. N., and Berger, F. (2005). Maternal control of integument cell elongation and zygotic control of endosperm growth are coordinated to determine seed size in Arabidopsis. Plant Cell 17 (1), 52-60. doi: $10.1105 /$ tpc.104.027136

Gifford, M. L., Dean, S., and Ingram, G. C. (2003). The Arabidopsis ACR4 gene plays a role in cell layer organisation during ovule integument and sepal margin development. Development 130 (18), 4249-4258. doi: 10.1242/ dev.00634

Haughn, G., and Chaudhury, A. (2005). Genetic analysis of seed coat development in Arabidopsis. Trends Plant Sci. 10 (10), 472-477. doi: 10.1016/ j.tplants.2005.08.005

Huang, J., Wijeratne, A. J., Tang, C., Zhang, T., Fenelon, R. E., Owen, H. A., et al. (2016). Ectopic expression of TAPETUM DETERMINANT1 affects ovule development in Arabidopsis. J. Exp. Bot. 67 (5), 1311-1326. doi: 10.1093/jxb/ erv523

Ingram, G. C. (2010). Family life at close quarters: communication and constraint in angiosperm seed development. Protoplasma 247 (3-4), 195-214. doi: 10.1007/s00709-010-0184-y

Kato, M., Tsuge, T., Maeshima, M., and Aoyama, T. (2019). Arabidopsis PCaP2 modulates the phosphatidylinositol 4,5-bisphosphate signal on the plasma membrane and attenuates root hair elongation. Plant J. 99 (4), 610-625. doi: $10.1111 /$ tpj.14226

Kieber, J. J., Rothenberg, M., Roman, G., Feldmann, K. A., and Ecker, J. R. (1993). CTR1, a negative regulator of the ethylene response pathway in Arabidopsis, encodes a member of the raf family of protein kinases. Cell 72 (3), 427-441. doi: 0092-8674(93)90119-B

Koornneef, M. (1990). Mutations affecting the testa color in Arabidopsis. Arabidopsis Inf. Services. 27, 1-4.

Kulich, I., Cole, R., Drdova, E., Cvrckova, F., Soukup, A., Fowler, J., et al. (2010). Arabidopsis exocyst subunits SEC8 and EXO70A1 and exocyst interactor $\mathrm{ROH} 1$ are involved in the localized deposition of seed coat pectin. New Phytol. 188 (2), 615-625. doi: 10.1111/j.1469-8137.2010.03372.x

Kunieda, T., Shimada, T., Kondo, M., Nishimura, M., Nishitani, K., and HaraNishimura, I. (2013). Spatiotemporal secretion of PEROXIDASE36 is required for seed coat mucilage extrusion in Arabidopsis. Plant Cell 25 (4), 1355-1367. doi: $10.1105 /$ tpc. 113.110072

Le, B. H., Cheng, C., Bui, A. Q., Wagmaister, J. A., Henry, K. F., Pelletier, J., et al. (2010). Global analysis of gene activity during Arabidopsis seed development and identification of seed-specific transcription factors. Proc. Natl. Acad. Sci. U. S. A. 107 (18), 8063-8070. doi: 10.1073/pnas.1003530107

Lepiniec, L., Debeaujon, I., Routaboul, J. M., Baudry, A., Pourcel, L., Nesi, N., et al. (2006). Genetics and biochemistry of seed flavonoids. Annu. Rev. Plant Biol. 57, 405-430. doi: 10.1146/annurev.arplant.57.032905.105252

Liljegren, S. J., Ditta, G. S., Eshed, Y., Savidge, B., Bowman, J. L., and Yanofsky, M. F. (2000). SHATTERPROOF MADS-box genes control seed dispersal in Arabidopsis. Nature 404 (6779), 766-770. doi: 10.1038/35008089

Loubery, S., De Giorgi, J., Utz-Pugin, A., Demonsais, L., and Lopez-Molina, L. (2018). A maternally deposited endosperm cuticle contributes to the 
physiological defects of transparent testa seeds. Plant Physiol. 177 (3), 12181233. doi: $10.1104 / p p .18 .00416$

Lu, P., Porat, R., Nadeau, J. A., and O’Neill, S. D. (1996). Identification of a meristem L1 layer-specific gene in Arabidopsis that is expressed during embryonic pattern formation and defines a new class of homeobox genes. Plant Cell 8 (12), 2155-2168. doi: 10.1105/tpc.8.12.2155

Lurin, C., Andres, C., Aubourg, S., Bellaoui, M., Bitton, F., Bruyere, C., et al. (2004). Genome-wide analysis of Arabidopsis pentatricopeptide repeat proteins reveals their essential role in organelle biogenesis. Plant Cell 16 (8), 2089-2103. doi: $10.1105 /$ tpc.104.022236

Martin, A., Troadec, C., Boualem, A., Rajab, M., Fernandez, R., Morin, H., et al. (2009). A transposon-induced epigenetic change leads to sex determination in melon. Nature 461 (7267), 1135-1138. doi: 10.1038/nature08498

Meyer, H. M., Teles, J., Formosa-Jordan, P., Refahi, Y., San-Bento, R., Ingram, G., et al. (2017). Fluctuations of the transcription factor ATML1 generate the pattern of giant cells in the Arabidopsis sepal. Elife 6. doi: 10.7554/eLife.19131

Mizzotti, C., Mendes, M. A., Caporali, E., Schnittger, A., Kater, M. M., Battaglia, R., et al. (2012). The MADS box genes SEEDSTICK and ARABIDOPSIS Bsister play a maternal role in fertilization and seed development. Plant J. 70 (3), 409420. doi: 10.1111/j.1365-313X.2011.04878.x

Mizzotti, C., Ezquer, I., Paolo, D., Rueda-Romero, P., Guerra, R. F., Battaglia, R., et al. (2014). SEEDSTICK is a master regulator of development and metabolism in the Arabidopsis seed coat. PloS Genet. 10 (12), e1004856. doi: 10.1371/journal.pgen.1004856

Nesi, N., Debeaujon, I., Jond, C., Stewart, A. J., Jenkins, G. I., Caboche, M., et al. (2002). The TRANSPARENT TESTA16 locus encodes the ARABIDOPSIS BSISTER MADS domain protein and is required for proper development and pigmentation of the seed coat. Plant Cell 14 (10), 2463-2479. doi: 10.1105/tpc.004127

Pinyopich, A., Ditta, G. S., Savidge, B., Liljegren, S. J., Baumann, E., Wisman, E., et al. (2003). Assessing the redundancy of MADS-box genes during carpel and ovule development. Nature 424 (6944), 85-88. doi: 10.1038/nature01741

Ranocha, P., Francoz, E., Burlat, V., and Dunand, C. (2014). Expression of PRX36, PMEI6 and SBT1.7 is controlled by complex transcription factor regulatory networks for proper seed coat mucilage extrusion. Plant Signal Behav. 9 (11), e977734. doi: 10.4161/15592324.2014.977734

Rodrigues-Pousada, R. A., De Rycke, R., Dedonder, A., Van Caeneghem, W., Engler, G., Van Montagu, M., et al. (1993). The Arabidopsis 1Aminocyclopropane-1-Carboxylate Synthase Gene 1 Is Expressed during Early Development. Plant Cell 5 (8), 897-911. doi: 10.1105/tpc.5.8.897

Roszak, P., and Kohler, C. (2011). Polycomb group proteins are required to couple seed coat initiation to fertilization. Proc. Natl. Acad. Sci. U. S. A. 108 (51), 20826-20831. doi: 10.1073/pnas.1117111108

Saez-Aguayo, S., Ralet, M. C., Berger, A., Botran, L., Ropartz, D., Marion-Poll, A., et al. (2013). PECTIN METHYLESTERASE INHIBITOR6 promotes Arabidopsis mucilage release by limiting methylesterification of homogalacturonan in seed coat epidermal cells. Plant Cell 25 (1), 308-323. doi: $10.1105 /$ tpc.112.106575

Sagasser, M., Lu, G. H., Hahlbrock, K., and Weisshaar, B. (2002). A. thaliana TRANSPARENT TESTA 1 is involved in seed coat development and defines the WIP subfamily of plant zinc finger proteins. Genes Dev. 16 (1), 138-149. doi: $10.1101 / \operatorname{gad} .212702$

Salminen, T. A., Blomqvist, K., and Edqvist, J. (2016). Lipid transfer proteins: classification, nomenclature, structure, and function. Planta 244 (5), 971-997. doi: $10.1007 / \mathrm{s} 00425-016-2585-4$
San-Bento, R., Farcot, E., Galletti, R., Creff, A., and Ingram, G. (2014). Epidermal identity is maintained by cell-cell communication via $a$ universally active feedback loop in Arabidopsis thaliana. Plant J. 77 (1), 46-58. doi: 10.1111/ tpj. 12360

Schneider, C. A., Rasband, W. S., and Eliceiri, K. W. (2012). NIH Image to Image): 25 years of image analysis. Nat. Methods 9 (7), 671-675. doi: 10.1038/ nmeth.2089

Schneitz, K., Hulskamp, M., and Pruitt, R. E. (1995). Wild-type ovule development in Arabidopsis thaliana: a light microscope study of cleared whole-mount tissue. Plant J. 7 (5), 731-749. doi: 10.1046/j.1365-313X.1995.07050731.x

Sessions, A., Weigel, D., and Yanofsky, M. F. (1999). The Arabidopsis thaliana MERISTEM LAYER 1 promoter specifies epidermal expression in meristems and young primordia. Plant J. 20 (2), 259-263. doi: 10.1046/j.1365313x.1999.00594.x

Smyth, G. K. (2004). Linear models and empirical bayes methods for assessing differential expression in microarray experiments. Stat. Appl. Genet. Mol. Biol. 3, Article3. doi: 10.2202/1544-6115.1027

Storey, J. D., and Tibshirani, R. (2003). Statistical significance for genomewide studies. Proc. Nat. Acad. Sci. U. S. A. 100 (16), 9440-9445. doi: 10.1073/ pnas. 1530509100

Tsai, A. Y., Kunieda, T., Rogalski, J., Foster, L. J., Ellis, B. E., and Haughn, G. W. (2017). Identification and characterization of arabidopsis seed coat mucilage proteins. Plant Physiol. 173 (2), 1059-1074. doi: 10.1104/pp.16.01600

Voiniciuc, C., Schmidt, M. H., Berger, A., Yang, B., Ebert, B., Scheller, H. V., et al. (2015). MUCILAGE-RELATED10 produces galactoglucomannan that maintains pectin and cellulose architecture in arabidopsis seed mucilage. Plant Physiol. 169 (1), 403-420. doi: 10.1104/pp.15.00851

Wang, X., Zhu, L., Liu, B., Wang, C., Jin, L., Zhao, Q., et al. (2007). Arabidopsis MICROTUBULE-ASSOCIATED PROTEIN18 functions in directional cell growth by destabilizing cortical microtubules. Plant Cell 19 (3), 877-889. doi: $10.1105 /$ tpc. 106.048579

Xu, S. L., Rahman, A., Baskin, T. I., and Kieber, J. J. (2008). Two leucine-rich repeat receptor kinases mediate signaling, linking cell wall biosynthesis and ACC synthase in Arabidopsis. Plant Cell 20 (11), 3065-3079. doi: 10.1105/ tpc.108.063354

Xu, W., Fiume, E., Coen, O., Pechoux, C., Lepiniec, L., and Magnani, E. (2016). Endosperm and nucellus develop antagonistically in arabidopsis seeds. Plant Cell 28 (6), 1343-1360. doi: 10.1105/tpc.16.00041

Yang, Y. H., Dudoit, S., Luu, P., Lin, D. M., Peng, V., Ngai, J., et al. (2002). Normalization for cDNA microarray data: a robust composite method addressing single and multiple slide systematic variation. Nucleic Acids Res. 30 (4), e15. doi: 10.1093/nar/30.4.e15

Conflict of Interest: The authors declare that the research was conducted in the absence of any commercial or financial relationships that could be construed as a potential conflict of interest.

Copyright $\odot 2020$ Coen, Lu, Xu, Pateyron, Grain, Péchoux, Lepiniec and Magnani. This is an open-access article distributed under the terms of the Creative Commons Attribution License (CC BY). The use, distribution or reproduction in other forums is permitted, provided the original author $(s)$ and the copyright owner(s) are credited and that the original publication in this journal is cited, in accordance with accepted academic practice. No use, distribution or reproduction is permitted which does not comply with these terms. 